\title{
Electric Vehicle Smart Charging Reservation Algorithm
}

\author{
Radu Flocea 1, Andrei Hîncu 1, Andrei Robu 1, Stelian Senocico 1, Andrei Traciu 1, Remus Marian Baltariu 1, Con- \\ stantin Filote $^{2^{*}}$ and Maria Simona Răboacă ${ }^{2,3}$ \\ 1 Research and Development Department, ASSIST Software, Str. Tipografiei Nr.1, 720043 Suceava, Romania; \\ radu.flocea@assist.ro, andrei.hincu@assist.ro, andrei.robu@assist.ro, stelian.senocico@assist.ro, an- \\ drei.traciu@assist.ro, remus.baltariu@assist.ro \\ 2 Faculty of Electrical Engineering and Computer Science, Stefan cel Mare University of Suceava, Str. Univer- \\ sitatii Nr.13, 720229 Suceava, Romania; filote.constantin@gmail.com, simona.raboaca@icsi.ro \\ 3 National Research and Development Institute for Cryogenic and Isotopic Technologies-ICSI Rm. Valcea, \\ 240050 Ramnicu Valcea, Romania; simona.raboaca@icsi.ro \\ * Correspondence: simona.raboaca@icsi.ro
}

\begin{abstract}
The widespread adoption of electromobility constitutes one of the measures designed to reduce air pollution caused by traditional fossil fuels. However, several factors are currently impending this process, ranging from insufficient charging infrastructure, battery capacity, long queueing and charging time, to psychological factors. On top of range anxiety, the frustration of the EV drivers is further fueled by the lack the uncertainty of finding an available charging point on their route. To address this issue, we propose a solution that comes to bypass the limitations of the Reserve now function of the OCPP standard, enabling drivers to make charging reservations for the upcoming days, especially when planning a longer trip. We created an algorithm that generates reservation intervals based on the charging station's reservation and transaction history. Subsequently, we ran a series of test cases that yielded promising results, with no overlapping reservations.
\end{abstract}

Keywords: smart scheduling; smart reservations; reinforcement learning; electric vehicle charging; electric vehicle charging management platform; neural network; DQN reinforcement learning algorithm

\section{Introduction}

Over the last few decades, there has been an alarming increase in the unsustainable exploitation of natural resources and environmental degradation. Nonetheless, a shift toward environmental protection has been registered, with regulation policies being enforced and public awareness steadily increasing.

On a global scale, we are currently witnessing a transition towards low-emission mobility, and it is safe to assume that this process is accelerating. Considering the Paris Agreement, the 2030 Agenda for Sustainable Development and the European Strategy for Low-Emission Mobility [1], governments and industry manufacturers have begun to take consistent steps in implementing these regulations and transitioning to an electro-mobility concept and way of life. Nonetheless, there are several factors that hinder the largescale adoption of electric vehicles $(\mathrm{EV})$, from the purchasing costs, the battery operating range, insufficient charging infrastructure and long charging times to psychological factors. When studying the challenges and opportunities in adopting electric vehicles, Faizal et al. [2] identified range anxiety, the long queuing time, and the uncertainty of finding an available charging point, when necessary, as some of the factors that negatively impact the consumers' purchasing intensions.

In this context, we propose a solution to enable the EV owner to create charging station reservations for upcoming days, especially for longer trips that require multiple charging operations, and to ensure a positive user experience that will ultimately stimulate EV adoption. To this end, we have structured the present paper into several sections. 
Following the introduction, the second chapter constitutes a brief overview of the current state of the art concerning the use of reservation algorithms as an extension of the OCPP protocol. After having presented the work methodology, we thoroughly describe the custom reservation algorithm and its advantages, compared with the existing OCPP reservation possibilities. To further illustrate the algorithm workflow, we have provided, in section 5, an overview of the Smart EVC platform case study. Finally, we analysed the results, stated the conclusion we reached after having conducted this study, as well as future work envisaged to take the proposed solution one step further.

\section{State of the Art}

As stated in the World Economic Forum's January 2018 report, Electric Vehicles for Smarter Cities: The Future of Energy and Mobility [3], radical sustainable and secure mobility and energy solutions are required to prevent congestion and pollution in urban areas that have already been and will continue to be reshaped by demographic shifts. The authors emphasize that the deployment of charging infrastructure should be based on the long-term mobility transformation. Furthermore, they believe that reducing range anxiety and developing smart charging technologies are critical components of the EV market approach, as they will contribute to the adoption of electro-mobility.

One of the major drawbacks of adopting electromobility, according to Ruzmetov et al. [4], is the EV driver's lack of certainty that there will be an available charging point once they reach the charging station on their route. The authors present a platform designed to ensure continuous cooperation among the various entities involved: energy suppliers, charging stations, EVs, and EV users, proposing an optimization of EV scheduling and allocation to charging stations. When proposing a charging station, the driver's destination, as well as the battery level are considered to ensure that they are not diverted from the route.

Wang et al [5], too, underline the importance of a scheduling strategy to fill the gap between EV charging requirements and the resources provided by the charging stations and to ensure a positive user experience that would encourage EV adoption.

Qin and Zhang [6] conducted a theoretical study that enabled them to develop a distributed scheduling protocol intended to reduce waiting time, which includes both queuing time and actual charging time, during a trip along a highway. The reservations made by EV drivers for their next charging, as well as the reservation adjustments, are based on the minimum waiting time communicated by charging stations, which update this information on a regular basis to allow drivers to make the best choice in terms of waiting time.

Park et al. [7] introduce algorithms for the recommendation of charging stations along a route, ensuring three alternatives for the user, depending on the envisaged final battery level and waiting time.

Cao et al. [8] sought to address a series of issues related to the charging management of EVs travelling longer distances, while ensuring efficiency, scalability, and coordination. The authors propose a publish/subscribe communication system for enabling fast charging, in which network entities share relevant information such as charge point availability and reservations made by the drivers. Furthermore, to coordinate EV drivers' charging arrangements, they have created a distributed charging management strategy, so as to limit the queuing time by selecting the most appropriate charging station.

In the same line, Hye-Jin et al. [9] propose a scheduling system relying on reservations made by the users, with the main goal of increasing EV user satisfaction by reducing both costs and queuing times, while maximizing charging station utilization. A linear ranking function is constructed around several factors such as estimated time of arrival of the EVs, waiting time and the energy requirements, in order to facilitate the scheduling process. Badmadjian et al. [10] suggest that reservation schemes could constitute a reliable solution for the challenges faced by the EV industry and infrastructure providers. They propose an architecture for interoperable reservation systems that considers needs of the main network entities involved in the process. 
Raboaca et al. [11] also investigated the environmental benefits of electric vehicles and proposed software solutions to encourage the adoption of electric vehicles by providing greater control over the EV charging process. Furthermore, the article provided an OCPP (Open Charge Point Protocol) specifications analysis, underlining that the charging process could be improved through the integration of a reservation feature. OCPP is an open standard that enables communication between electric vehicles, charging stations and a central management system, regardless of manufacturer features, and ensures management of all factors and entities involved in the recharging operation. [12] This has ensured that EV networks are open and accessible. As a result, end users benefit from flexibility and the ability to choose which network or charging station they use. The main purpose of OCPP is to ensure the interoperability of the EV charging infrastructure, characterized by versatility and simplicity in terms of use for EV drivers and system administrators, alike [13]. Currently, OCPP 1.6 does not support scheduling the reservation of a connector for a future date, being able to reserve only at the exact moment the user makes the reservation. The reservation is terminated either when the user with the associated idTag arrives at the charging point, the expiry time is reached, or the user cancels the reservation [14].

Oricioni and Conti [15] propose an extension of the OCPP standard that would allow the user to participate in the charging optimization process, introducing an advance reservation option. The authors also point out that OCPP standard does not enable reservations made in advance for a later moment and consider that the underlying reasons for this shortcoming might reside in the complexity of this type of scheduling and the firstcome-first-served approach, in the detriment of those with remote reservations

\section{Methodology}

While conducting research in view of developing the Smart EVC platform - an intelligent charging station management platform based on Blockchain and Artificial Intelligence - the methodology for the case study in the current paper has been gradually improved, based on the literature (WOS, Science Direct, Scopus) and on the results and feedback received after having attended several conferences in the field.

Therefore, we envisaged the case where several EV drivers were subjected to long queuing times due to the OCPP reservation protocol that allows for overlapping reservations. To address this issue, we developed an algorithm that generates reservation intervals based on coefficients obtained from the charging station's reservation and transaction history. We opted to implement the reservation feature as a generic method of planning a reservation for all types of electric vehicles in which drivers are familiar with their vehicles' specifications and can easily estimate charging time. We performed a series of test cases that yielded promising results, overlapping reservations being completely avoided.

\section{Custom Reservation Algorithm}

As pointed out above, there are several limitations to the OCPP reservation feature, where a reservation is initiated when the driver creates it and is valid until a specified end time, blocking the selected connector for other drivers, except for the one that created the reservation. The reservation lifetime ends when the user starts their transaction [14].

Making this approach possible and following the OCPP path also implies a lot of changes and unnecessary refactoring in the already working code in the Web API for reservations and transactions. Thus, below will present all the downsides of this approach and the benefits of the custom reservations algorithm.

We opted for the implementation of the reservations in Web API because this grants us more control over them. Moreover, the reservation in our system, which will be further developed in the Use case section, is made up of both the time span selected by the driver and time calculated by the algorithm and added to the initial user-set StartTime. Furthermore, this kind of reservation can be made in advance and can be successfully used in the Plan a trip feature, which provides the user with advance information related to the 
charging needs and options along the route, enabling them to make reservations for the upcoming trip. To better illustrate the flexibility of this type of reservation, we should underline that it can be easily manipulated by changing the status of the reservation and the connector, according to the user's activity. Furthermore, a transaction can be automatically interrupted when the reservation comes to an end.

For a better understanding of the differences between the OCPP Reservations and the proposed custom reservation algorithm, we can study the following plot diagrams:

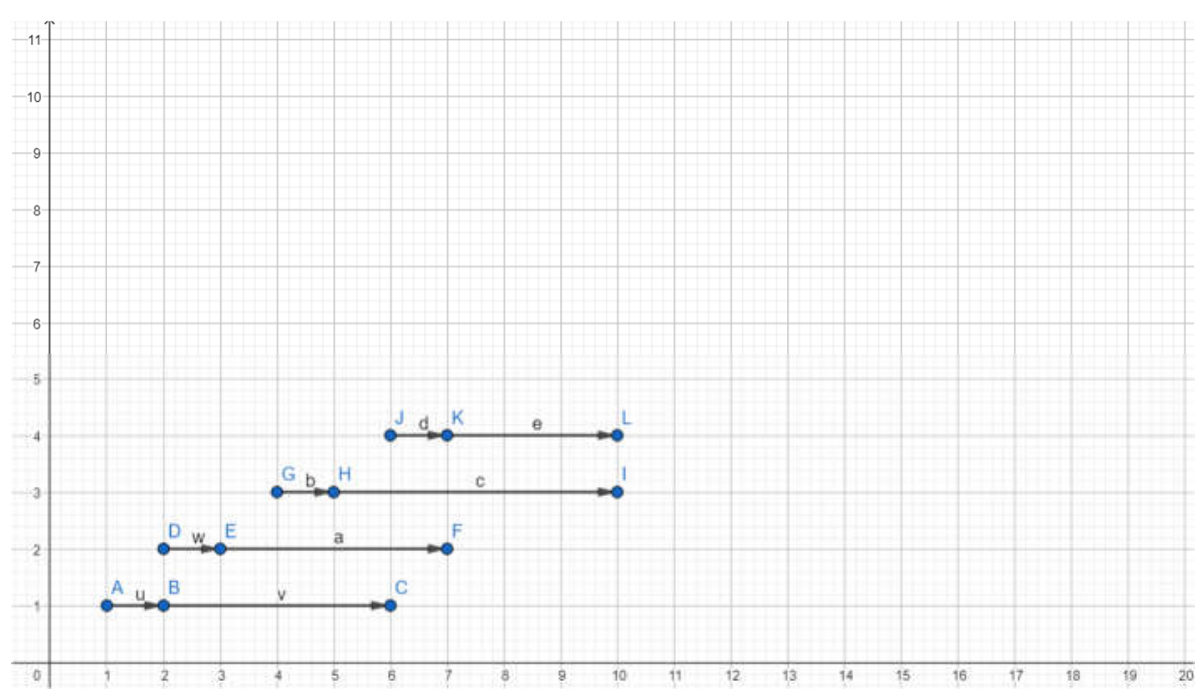

Figure 1. OCPP Reserve now.

For this diagram, the $\mathrm{X}$ axis represents a full day timeline, the $\mathrm{Y}$ axis is used just for a better view and convenience. As mentioned before, in OCPP, the reservation time span is limited to the time before the charging process starts. In our case illustrated above, the $\mathrm{AB}, \mathrm{DE}, \mathrm{GH}$ and JK segments constitute the reservations. None of the reservations overlap with one another, and this might be considered an optimal solution. Nonetheless, as the users have no limitations imposed by the OCPP reservation, they can block the charging point for as long as they need, and therefore, their charging time is likely to overlap with other reservations. Consequently, this will generate confusion among the other users that made a reservation, and the queueing time will automatically increase.

Alternatively, by using the proposed custom Reservation Algorithm, the diagram will look like this:

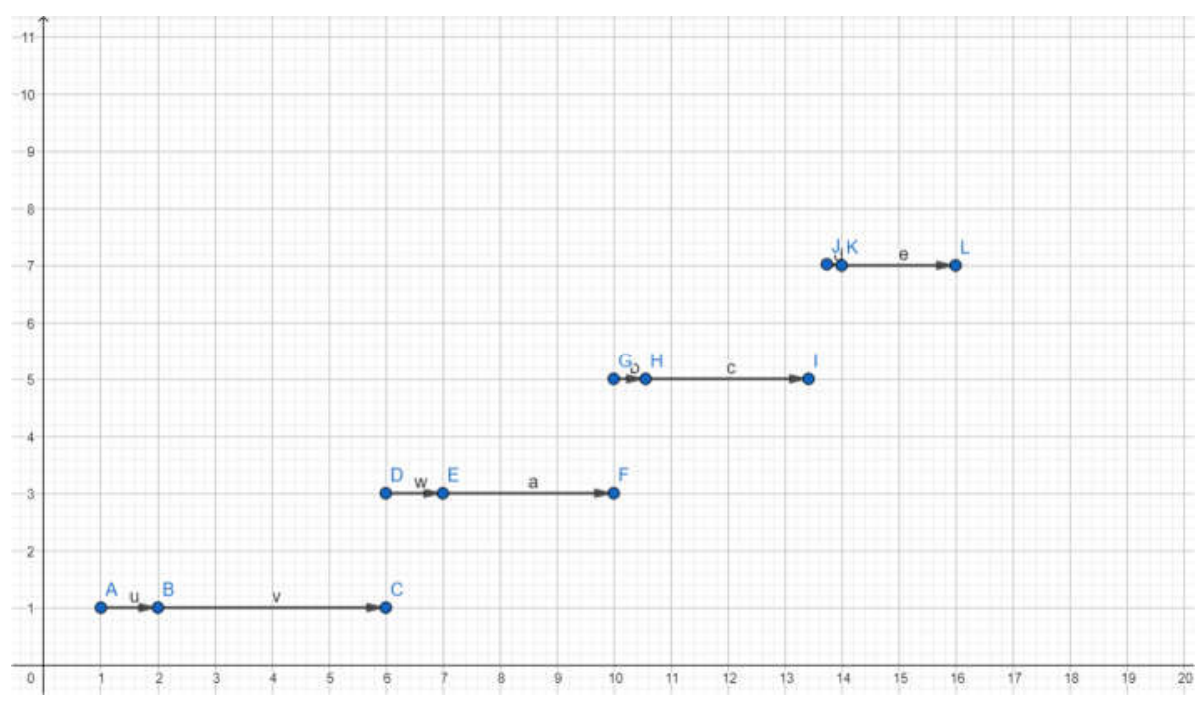

Figure 2. Custom Reservation Algorithm Reservation. 
With the custom reservation algorithm, the reservation time is made up of the extra time prior to the charging process and the actual charging time. Therefore, the reservation of the connector starts at the time selected by the user and ends when the user interrupts the charging process and leaves the station. In this case, there will be no overlaps or uncertainties because the reservation guarantees the availability of the charging point, preventing other users from blocking it during the reservation period. This constitutes the main advantage of the proposed custom Reservation Algorithm, in comparison with the OCPP Reservations.

To further explain the way in which the proposed custom algorithm works, we have provided below a detailed description of the process.

When making a reservation, the user must select a valid time slot, during which other EV owners will be prevented from charging their vehicles to the reserved charging point. Several status types have been assigned to a charging point reservation: it is New, upon creation and then it turns to In progress when the reservation interval starts and to Finished at the end of the time slot. If the user cancels the reservation, its status becomes Cancelled.

Before making a reservation, the user must be assured that no other EVs are or will be plugged to the connector they are about to reserve. For this reason, each reservation is processed by an algorithm designed to determine the best possible StartTime. This algorithm considers several factors and, as a result, will return a new reservation with additional time added to the user specified StartTime. In the current version, this additional time can be as little as 0 hours and as much as 4 hours, and it is calculated based on several factors, as follows:

1. All the transactions made in the previous two weeks on the charging point the user is about to reserve are retrieved from the database, being the only data relevant for the algorithm at this point.

2. Next, two coefficients influencing the output of the algorithm are calculated.

The first one is the connectorRequestCoefficient, which determines the exploitation degree of the connector (charging point), comparing the values from the previous week and two weeks prior. This coefficient influences $50 \%$ of the result of the algorithm, and is calculated using to the following formula:

connectorRequestCoefficient = lastTwoWeeksTransactions;

(double)lastWeekTransactions / where lastWeekTransactions represents the number of transactions recorded in the previous week on the connector reserved by the user and lastTwoWeeksTransactions returns the number of transactions registered during the last two weeks. According to the formula, this coefficient can range from 1 to 0 . More precisely, it can have the minimum value 0 if in the last week there were no transactions on that charging point and all the transactions on that connector occurred only two weeks prior. The maximum value 1 will be returned if all the transactions on that specific charging point occurred in the previous week.

The second coefficient is called overlappingCoefficient and it illustrates the utilization degree associated with the time slot selected by the user. It is calculated similarly to the first coefficient: having the data on the transactions performed in the previous two weeks, we determine how many occurred in a period overlapping the time slot selected by the user. To this end, we created the isOverlapping method that determines if two time slots have at least one point in common. The method returns the minimum value 0 in case none of the transactions made on the reserved connector in the last two weeks intersects with the selected time slot and the maximum value 1 if all the transactions overlap with this period. This coefficient has a $50 \%$ weight in the result of the algorithm.

3. Having made all the calculations and retrieved the connectorRequestCoefficient and the overlappingCoefficient, the final coefficient will be calculated using the formula below, which assigns the same weight to the first two coefficients: 


\section{$0.5 *$ connectorRequestCoefficient $+0.5 *$ overlappingCoefficient.}

This final coefficient has a value ranging from 0 to 1 and determines how long the reservation will be extended for. The number of extra hours that will be added to the reservation StartTime will be calculated as illustrated below:

\section{hoursToAdd = finalCoefficient ${ }^{*}$ maxExtraHours;}

We have considered that, in the process, a reservation can be moved from one day to another: for instance, if the reservation is initially made for 01:00 a.m. and the algorithm concludes that two extra hours are necessary, then the reservation will be extended, starting from 11:00 p.m., the previous day.

The reservation process is graphically illustrated below, in Figure 3.

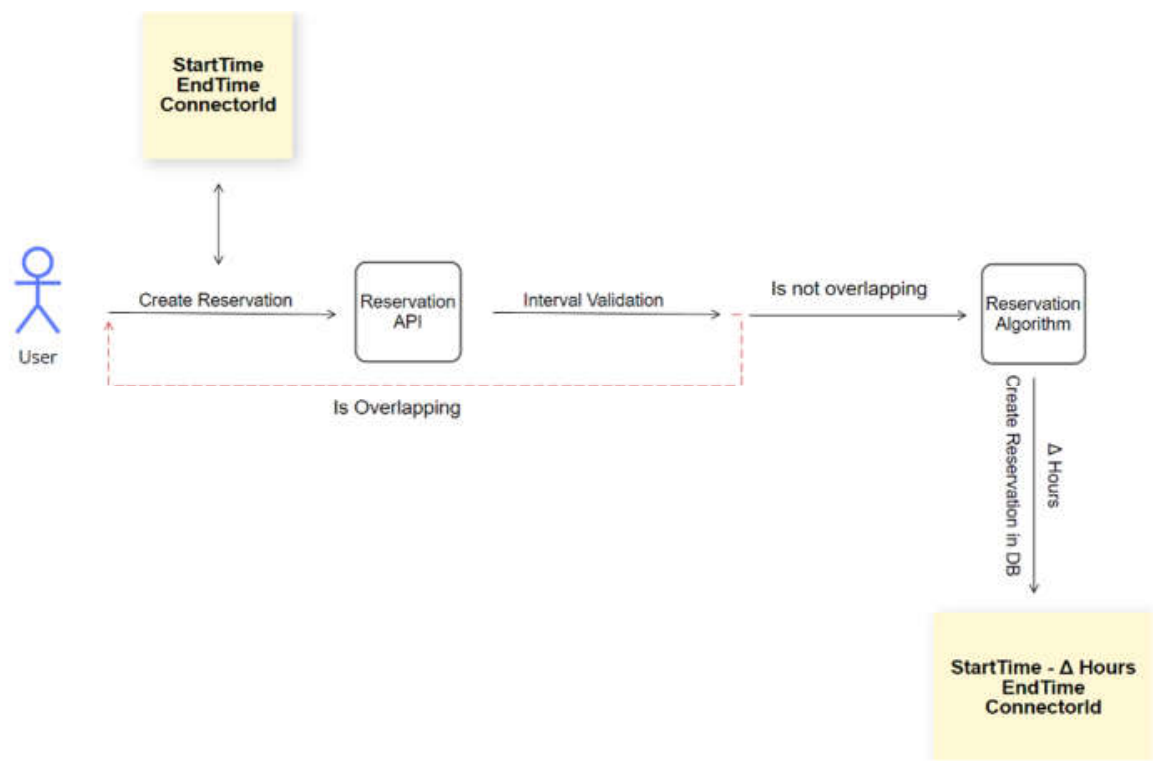

Figure 3. Reservation process.

\section{Case Study}

The proposed algorithm has been integrated in the Smart EVC solution - an intelligent charging station management platform based on Blockchain and Artificial Intelligence that allows users to interact with charging stations. The platform brings together various techniques for connecting electric vehicles to charging stations and the embedded Blockchain technology allows for the unification of payment systems for charging electric vehicles.

The mobile app developed within the Smart EVC project will be available on Android and iOS and will enable users to make reservations at the charging stations registered in the system. Using various pre-established parameters, the mobile application facilitates the users' interactions with charging stations and generates intelligent alerts on upcoming charging options.

To enable users to identify and get directions to available charging stations in their vicinity, the Maps SDK for Android and iOS has been integrated and the Directions and Places APIs from the Google console have been activated.

Upon authentication, the users will be directed to the MAP screen. The interaction with the map has been enabled through the LocationListener, GoogleMap.OnMarkerClickListener functions of the Google Maps SDK interfaces. 


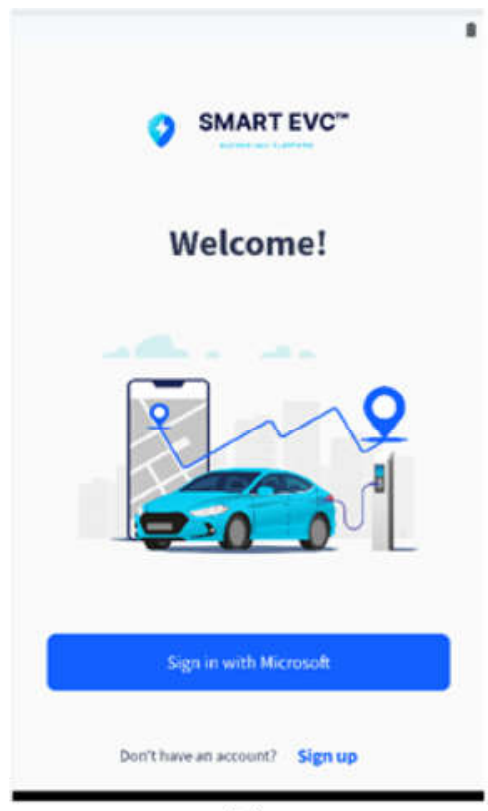

(a)

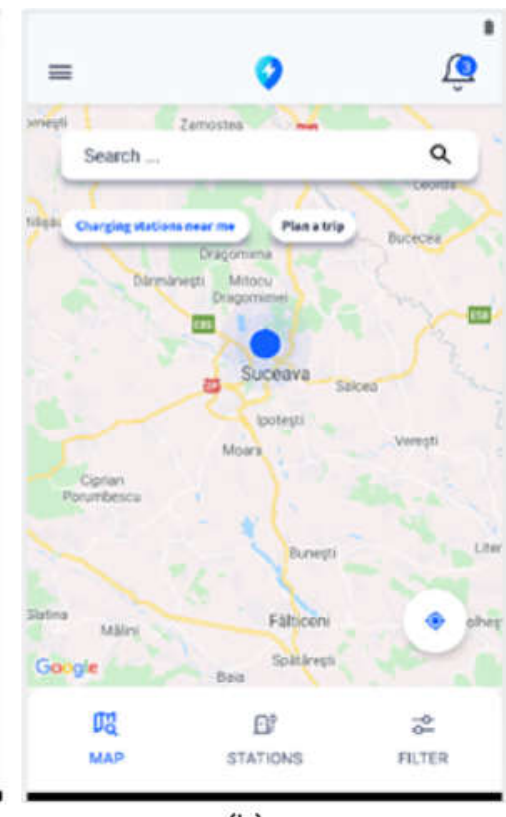

(b)

Figure 4. Smart EVC login and first page.

By clicking the Charging stations near me button, the stations corresponding to the options set by the user in the Filter section of the mobile app will be displayed, as illustrated in the images below. The user will click on a marker on the map to view the full details of a charging station: address, distance to the location, available connectors.

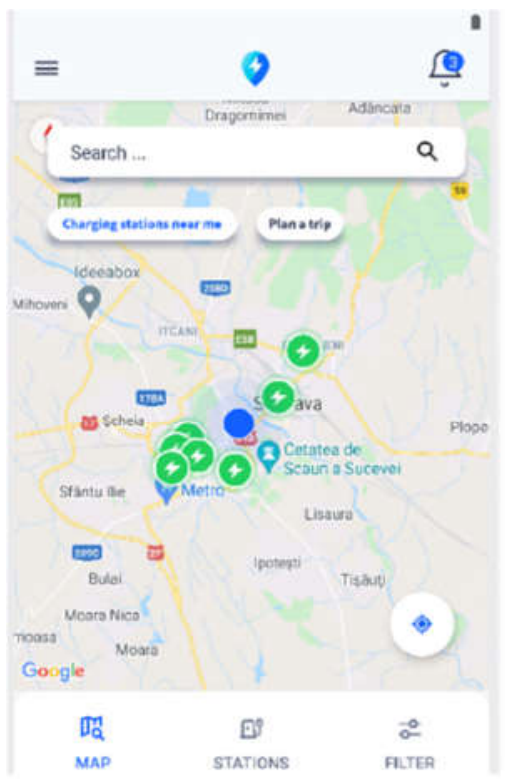

(a)

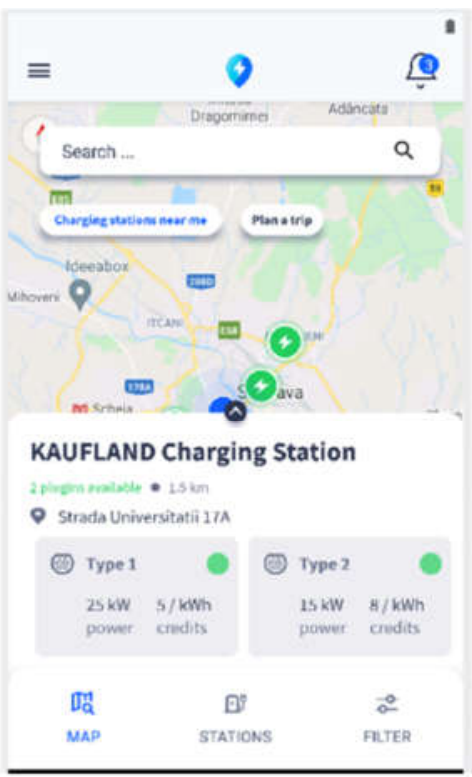

(b)

Figure 5. Smart EVC - Charging stations in the vicinity.

Moreover, the user can then expand the view of the station, gaining access to the full functionalities of a station: directions to the station, buttons to initiate the charging process or to make a reservation. 


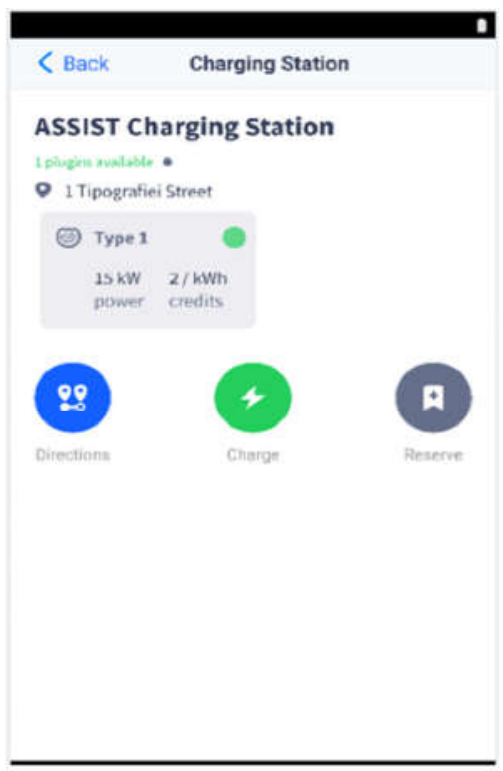

(a)

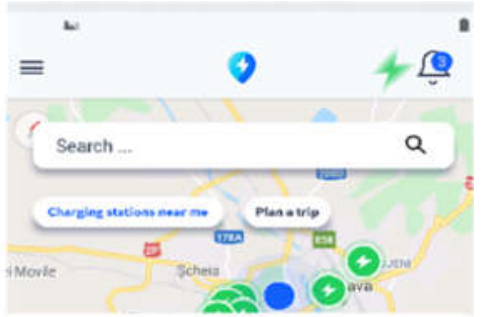

ASSIST Charging Station

1 1 ipogrefiei Street

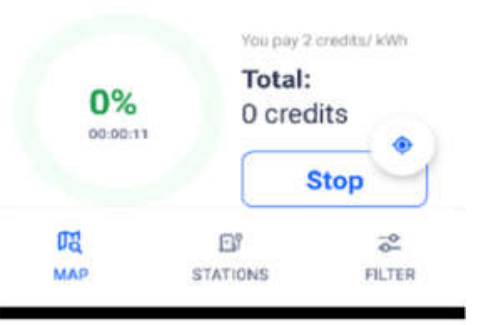

(b)

Figure 6. Smart EVC - Charging station.

When the user reaches the station and initiates the charging process, the API called SmartEvcOcppAPI sends other users the busy status, until the charging is complete. The Start / Stop charging transactions are enabled through the unique charging point identifiers: chargingStationId, connectorId, organizationId.

The Plan a trip section has been created, to assist users in planning their trip, especially for longer routes that require several charging operations, providing the option to make reservations in advance.

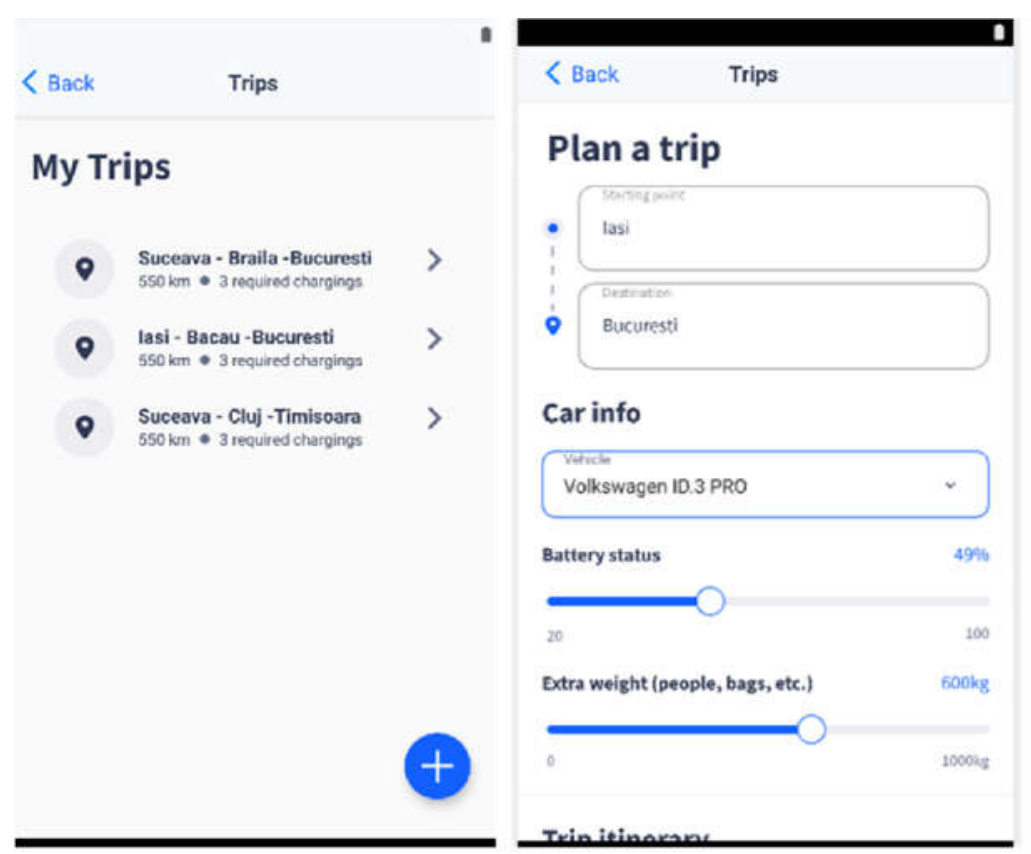

(a) (b)

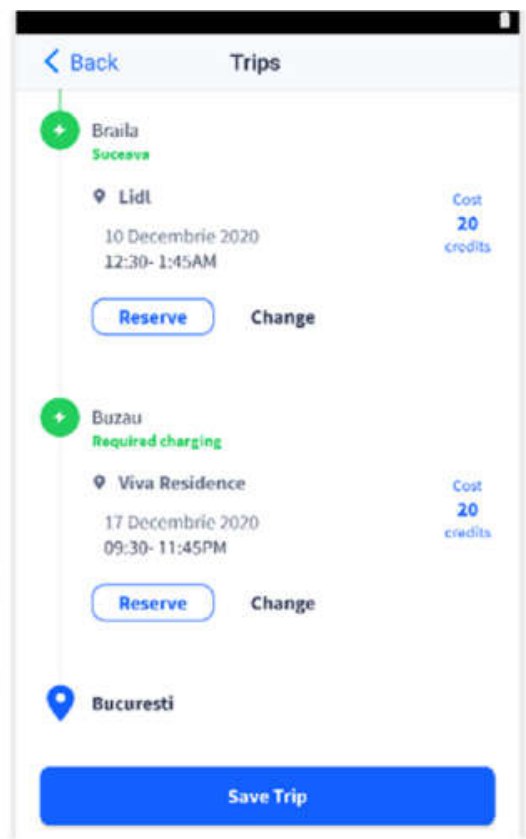

(c)

Figure 7. Smart EVC - Plan a trip section.

The custom reservation algorithm presented in the current paper has been integrated in the Smart EVC platform, and the EV driver will engage with it when accessing the "Reserve station" page in the mobile application, illustrated in Figure 8. 


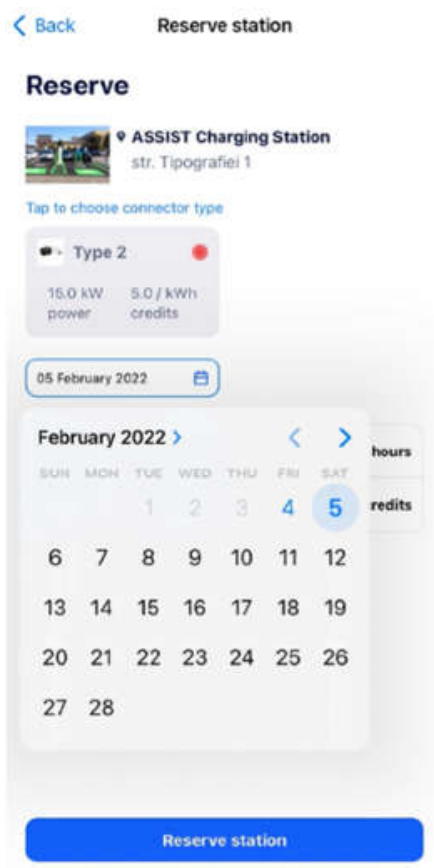

(a)

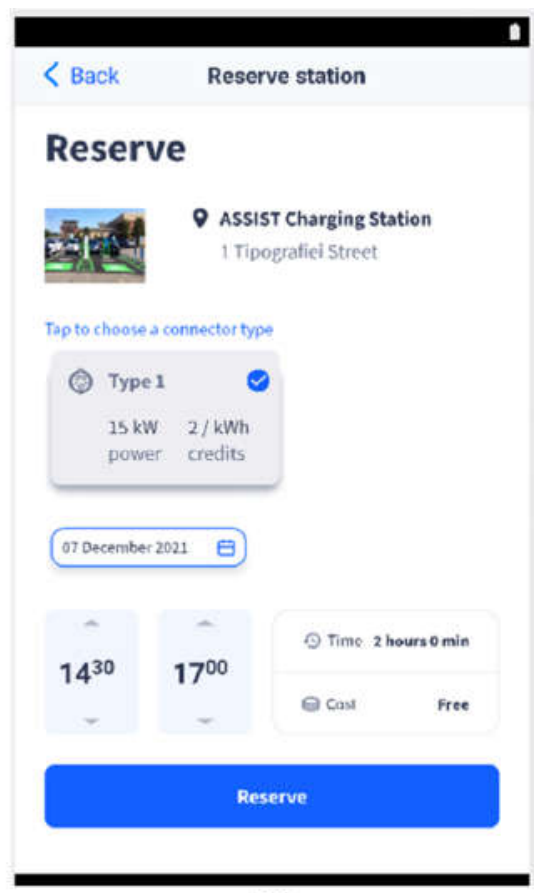

(b)

Figure 8. Smart EVC Reservations.

The users must select the charging station that they want to reserve, as well as a suitable connector for their electric vehicle. Next, they have to indicate the date of the reservation and finally, to select a time slot from all the available intervals that will be displayed on the screen.

The duration of the reservation and the cost will be automatically calculated as illustrated below:

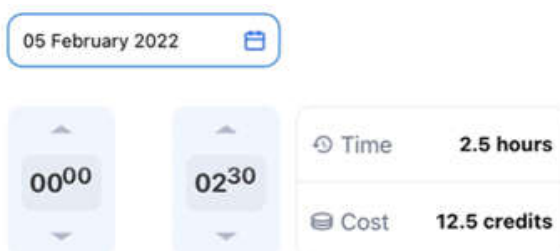

Figure 9. Smart EVC - Reservation duration and cost.

This is all the data requested from the users, and when they click on the "Reserve station" button, the reservation algorithm will be applied on the data provided by the user, and the StartTime will be adjusted based on all the important factors and statistics on the chosen station, connector, timeline etc.

The users are also able to view their future reservations and update a reservation if necessary (Fig. $10 \mathrm{a}$ and $10 \mathrm{~b}$ ). 


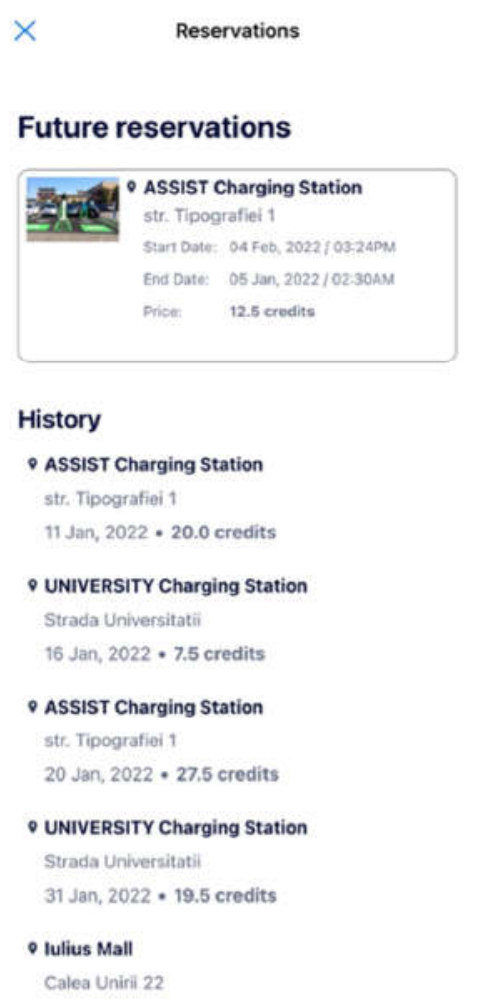

(a)

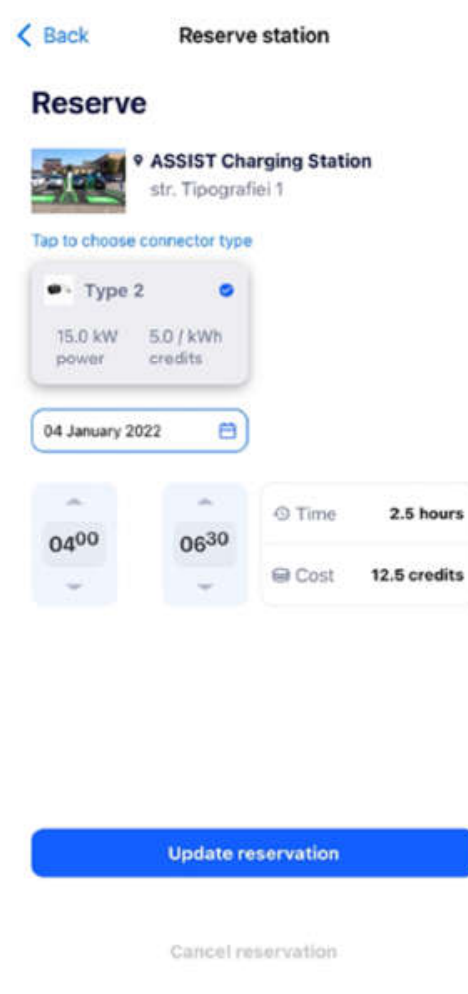

(b)

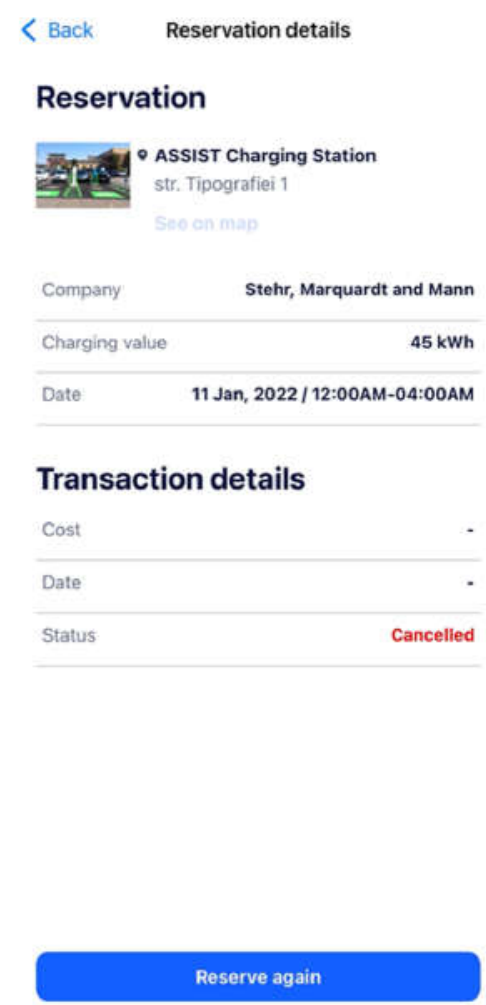

(c)

Figure 10. Smart EVC - Future reservations visualization.

If the user updates their reservation with a new set of data, by clicking the "Update reservation" button (Fig. 10b), the Reservation Algorithm will be called upon one more time and it will recalculate the response based on the newly entered data.

All the actions that are performed by the driver are used for the statistics, to ensure a better estimation and user experience when using the Reservation Algorithm.

The user is also allowed to cancel a reservation (Fig. 10b). This action will release the occupied time slot from the reservation history and will also update the statistics used for other upcoming reservations. The reservation history will enable users to reserve the same charge point repeatedly (Fig. 10c). This feature is convenient especially if the user travels on the same route frequently.

\section{Results and discussions}

We performed several test cases for the reservation algorithm where the connector of the station is reserved for the entire day and there were no overlaps. Even if the station is disconnected and comes back online, only the user that has the reservation at that moment can start a transaction. Another example of a test case is when a driver finishes a transaction before the reservation time ends, in which case, the connector would be released, so other drivers can charge until the next reservation time comes.

There are several external variables, though, that can influence the charging time and need to be further analysed, such as traffic jams, battery condition and energy consumption or weather conditions.

\section{- $\quad$ Traffic jams}

Research made in 2021 by "Which?", a United Kingdom organization that promotes informed consumer choice in the purchase of goods and services by testing products, has shown that in just over an hour and 15 minutes, $2 \%$ of battery from a $77 \mathrm{kWh}$ battery was discharged. This is the equivalent of 13 kilometers of range, considering it was on a summer day, but it is a well-known fact that cold weather will have a bigger impact on the car's power usage. [11] 
- $\quad$ Battery condition

When the battery is new, it seems to last forever and charge fast, but over time and use, their capacity will reduce, as well as the charging time. Even if the loss is not that big and is only around $2.3 \%$ per year, it is still significant when considering a charging reservation. [12]

- Weather

A study on electric vehicles charging in cold temperatures has shown that drivers still face some charging issues. The reason is that cold temperatures have a significant impact on the whole charging process because of the electrochemical reactions, and the management system will limit the charging rate, to avoid battery damage. This is the reason for which there will be inevitable alterations in the charging time. [13]

- $\quad$ Battery energy consumption

The energy consumption is defined in terms of kilowatt hour per one hundred kilometers, and it will obviously differ on a case-by-case basis and depending on many other factors such as driver's behavior or interior temperature regulation, but in the end, it will optimize or weaken the electricity consumption, which will certainly affect the charging time as well. [14]

- Conclusions and limitations

We have developed and trained a neural network that uses a history of the situations encountered thus far to identify the optimal charging station and time interval for recharging. Rewards are assigned to each decision made by the algorithm. In lack of available datasets, a simulator that generates training data has been implemented, creating new scenarios.

Because the training data is generated by a simulator, it rarely resembles real-world data. If the DQN algorithm encounters situations that differ significantly from the scenarios in the training data sets, it may make incorrect decisions. The considerable training time of the neural network constitutes yet another constraint, as it needs to go through multiple situations and iterations to reach an optimal solution. Furthermore, the training time is extended because every scenario must be simulated before the algorithm is assigned a reward.

\section{Conclusions}

In this article, we proposed a solution for the electric vehicle reservation challenge. We assumed that several users were waiting in a queue at a charging station with a single connector when following the OCPP reservation protocol that allowed for a lot of overlaps to occur. To implement a solution, we built an algorithm that creates reservation intervals based on coefficients provided from the reservation and transaction history of the specific charging station, ensuring consumer satisfaction. Also, we changed the reservation perspective by adding the charging time to it. To solve the overlap problem, every reservation can be controlled by the user that created it so no one else can start a transaction in the reservation interval. Moreover, when the reserved time ends the charging stops. By taking this into a real scenario there will be no overlaps.

\section{Future Research Directions}

To further expand the functionalities of the reservation module, we plan to add a new option where drivers can select a time span, and the application returns the closest charging points that are available at that moment.

To improve the algorithm, we intend to add a new method that generates an ideal reservation interval based on the current battery level and the level the user intends to reach at the end of the charging cycle. This involves taking into consideration all the influencing factors mentioned above and adding extra time to the reservation. 
Acknowledgments: This work was supported by a grant of the Romanian Ministry of Education and Research, CCCDI - UEFISCDI, project number PN-III-P2-2.1-PTE-2019-0642, within PNCDI III.

\section{References}

1. European Strategy for Low-Emission Mobility, available here: https://eurlex.europa.eu/resource.html?uri=cellar:e44d3c21-531e-11e6-89bd-01aa75ed71a1.0002.02/DOC_1\&format=PDF

2. Faizal, M., Feng, S. Y., Zureel, M. F., Sinidol, B. E., Wong, D., \& Jian, G. K. A REVIEW ON CHALLENGES AND OPPORTUNITIES OF ELECTRIC VEHICLES (EVS). Journal of Mechanical Engineering Research \& Developments, 42(4), 130-137. https://doi.org/10.26480/JMERD.04.2019.130.137 https://www.academia.edu/40020853/A_Review_on_Challenges_and_Opportunities_of_Electric_Vehicles_EVS_?email_w ork_card=view-paper

3. World Economic Forum, “Electric Vehicles for Smarter Cities: TheFuture of Energy and Mobility,”World Econ. Forum, no. January, p.32, 2018. https://www3.weforum.org/docs/WEF_2018_\%20Electric_For_Smarter_Cities.pdf

4. Ruzmetov, A., Nait-Sidi-Moh, A., Bakhouya, M., \& Gaber, J. (2013). Towards an optimal assignment and scheduling for charging electric vehicles. 2013 International Renewable and Sustainable Energy Conference (IRSEC). https://doi.org/10.1109/IRSEC.2013.6529691

5. Wang, R.; Chen, Z.; Xing, Q.; Zhang, Z.; Zhang, T. A Modified Rainbow-Based Deep Reinforcement Learning Method for Optimal Scheduling of Charging Station. Sustainability 2022, 14, 1884. https://doi.org/10.3390/su14031884

6. Qin, Hua \& Zhang, Wensheng. (2011). Charging scheduling with minimal waiting in a network of electric vehicles and charging stations. Proc. 8th ACM VANET. 51-60. 10.1145/2030698.2030706.

https://www.researchgate.net/publication/220926703_Charging_scheduling_with_minimal_waiting_in_a_network_of_elec tric_vehicles_and_charging_stations

7. Park, Chan \& Lee, Junghoon \& Park, Gyung \& Hyun, Jung. (2014). Development of Reservation Recommendation Algorithms for Charging Electric Vehicles in Smart-Grid Cities. International Journal of Smart Home. 8. 113-122. 10.14257/ijsh.2014.8.1.12.

8. Y. Cao et al., "Toward Efficient, Scalable, and Coordinated On-the-Move EV Charging Management," in IEEE Wireless Communications, vol. 24, no. 2, pp. 66-73, April 2017, doi: 10.1109/MWC.2017.1600254WC.

https://nrl.northumbria.ac.uk/id/eprint/30271/1/Towards\%20Efficient,\%20Scalable\%20and\%20Coordinated\%20On-themove\%20EV\%20Charging\%20Management.pdf

9. Kim HJ., Lee J., Park GL., Kang MJ., Kang M. (2010) An Efficient Scheduling Scheme on Charging Stations for Smart Transportation. In: Kim T., Stoica A., Chang RS. (eds) Security-Enriched Urban Computing and Smart Grid. SUComS 2010. Communications in Computer and Information Science, vol 78. Springer, Berlin, Heidelberg. https://doi.org/10.1007/978-3-642-16444-6_35

10. Basmadjian, R.; Kirpes, B.; Mrkos, J.; Cuchý, M. A Reference Architecture for Interoperable Reservation Systems in Electric Vehicle Charging. Smart Cities 2020, 3, 1405-1427. https://doi.org/10.3390/smartcities3040067 https://res.mdpi.com/d_attachment/smartcities/smartcities-03-00067/article_deploy/smartcities-03-00067-v2.pdf

11. Raboaca, MS, Meheden, M, Musat, A, et al. An overview and performance evaluation of Open Charge Point Protocol from an electromobility concept perspective. Int J Energy Res. 2022; 46( 2): 523- 543. doi:10.1002/er.7206

12. Chaoyue Zhao, Xiangdong You (2016) Research and Implementation of OCPP 1.6 Protocol: Proceedings of the 2017 2nd International Conference on Machinery, Electronics and Control Simulation (MECS 2017), Atlantis Press, Advances in Engineering Research https://doi.org/10.2991/mecs-17.2017.149

13. Venkata Pruthvi, Thota \& Dutta, Niladri \& Bobba, Phaneendra \& Vasudeva, B. (2019). Implementation of OCPP Protocol for Electric Vehicle Applications. E3S Web of Conferences. 87. 01008. 10.1051/e3sconf/20198701008. 
https://www.researchgate.net/publication/331281703_Implementation_of_OCPP_Protocol_for_Electric_Vehicle_Applicati ons

14. Online source: https://www.openchargealliance.org/

15. Orcioni, Simone \& Conti, Massimo. (2020). EV Smart Charging with Advance Reservation Extension to the OCPP Standard. Energies. 13. 3263. 10.3390/en13123263. https://www.mdpi.com/1996-1073/13/12/3263/pdf

16. Online source: https://www.which.co.uk/news/2021/08/electric-cars-in-traffic-jams-will-your-battery-cope

17. Online source: https://www.fleetnews.co.uk/electric-fleet/charging-and-infrastructure/make-your-electric-vehicle-batterylast-longer

18. Online source: https://eepower.com/news/quantifying-the-negative-impact-of-charging-evs-in-cold-temperatures

19. Online source: https://www.renaultgroup.com/en/news-on-air/news/everything-you-need-to-know-about-electric-carconsumption/ 